\title{
High-performance supercapacitors of hydrous ruthenium oxide/mesoporous carbon composites
}

\author{
Yaomin Zhao • Ling Liu • Juan Xu • Jie Yang • \\ Manming Yan $\cdot$ Zhiyu Jiang
}

Published online: 31 May 2006

(C) Springer-Verlag 2006

The online version of the original article can be found at: http://dx.doi. org/10.1007/s10008-006-0105-6

Unfortunately, the units of discharge capacity in Fig. 5 are wrongly given. The correct unit is $\mathrm{C} \mathrm{g}^{-1}$.

Y. Zhao $\cdot$ L. Liu $\cdot$ J. Xu $\cdot$ J. Yang $\cdot$ M. Yan $\cdot$ Z. Jiang $(\bowtie)$

Department of Chemistry, and Shanghai Key Laboratory of

Molecular Catalysis and Innovative Materials, Fudan University,

Shanghai 200433, People's Republic of China

e-mail: zyjiang@fudan.ac.cn 